\title{
Repeated Surgery for Recurrent Pseudoaneurysms after Thoracoabdominal Aortic Replacement
}

\author{
Kazuchika Suzuki $^{1}$, Naoyuki Ishigami ${ }^{1}$, Satoshi Akuzawa ${ }^{1}$, Abul Hasan Muhammad Bashar ${ }^{2}$, \\ Motoaki Shirakawa $^{1}$, Tatsuya Igarashi ${ }^{1}$, Hiroshi Mitsuoka ${ }^{3}$ \\ ${ }^{1}$ Fujieda Municipal General Hospital, Fujieda, Japan \\ ${ }^{2}$ National Institute of Cardiovascular Diseases Sher-E-Bangla, Nagar, Dhaka \\ ${ }^{3}$ Japan Red Cross Shizuoka Hospital, Shizuoka, Japan \\ Email: kazuchi@fj9.so-net.ne.jp
}

Received April 10, 2013; revised May 10, 2013; accepted May 18, 2013

Copyright (C) 2013 Kazuchika Suzuki et al. This is an open access article distributed under the Creative Commons Attribution License, which permits unrestricted use, distribution, and reproduction in any medium, provided the original work is properly cited.

\begin{abstract}
Aneurysm or pseudoaneurysm formation in the aortic patch containing the intercostal or visceral arteries is an unusual late complication after thoracoabdominal aortic surgery. We report the case of a 58-year-old woman who had previously undergone thoracoabdominal aortic replacement (Crawford extent II) for dissecting aneurysm. About 12 months after the operation repeated pseudoaneurysmal degenerations occurred at the intercostal or visceral artery reattachment site. They were repaired with open surgery or endovascular stent-graft. The patient recovered without major complications, and computed tomographic scans showed no recurrence of aneurysm or pseudoaneurysm at the sites of repair 1 year after the procedure.
\end{abstract}

Keywords: Thoracoabdominal Aortic Replacement; Pseudoaneurysm; Endovascular Stent-Graft

\section{Introduction}

Aneurysmal or pseudoaneurysmal degeneration of the intercostal artery (ICA) or visceral arterial patch (VAP) is an infrequent long-term complication of thoracoabdominal aortic aneurysm (TAAA) surgery [1-4]. Although open surgery has been applied for the re-reconstruction of ICA or visceral arteries, it is associated with considerable morbidity and mortality because of the invasive nature of the procedure. Recently the endovascular stent-graft (EVSG) techniques came to be used for such cases [5-9]. We describe an extremely rare case of recurrent VAP pseudoaneurysm following TAAA surgery that was treated with laparotomy and EVSG. To the best of our knowledge, there is no report describing the treatment of VAP aneurysm with a simple laparotomy.

\section{Case Report}

A 58-year-old female had an acute type B aortic dissection 14 years ago. She was managed conservatively and was being followed-up. As the descending aorta was gradually dilating, TAAA surgery (Crawford extent II) was performed with 4-branched aortic graft (VascuteckTerumo, Renfrewshire Scotland, UK) 3 years ago. The
T8 intercostal artery was reimplanted to the aortic graft as a small patch. The celiac axis (CA) and the superior mesenteric artery (SMA) were anastomosed to the first branch with an en-bloc technique, while the right and left renal arteries were individually reconstructed to the respective branches of the graft. The reconstructions of visceral arteries were performed with button techniques. Histopathological examination of the aortic wall revealed slight atherosclerotic changes without other pathognomonic findings. One year after TAAA surgery, the patient was admitted with severe back pain. Computed tomographic (CT) scan demonstrated a large hematoma in the retroperitoneal space and the patch containing the origins of CA and SMA was found to have aneurysmal dilatetion with extravascular leakage (Figures 1(a) and (b)). Emergency operation was carried out. Epigastric median laparotomy was performed and the CA, SMA and the prosthetic branch graft were exposed through the small omentum. To avoid ischemia of the perfusion area of the $\mathrm{CA}$, an 8Fr-catheter was inserted into the left gastric artery and connected to a $16 \mathrm{Fr}$-perfusion catheter that was inserted into the left femoral artery. The CA, SMA and the prosthetic graft were clamped near the aneurysmal aortic patch. The aortic patch was opened which revealed 


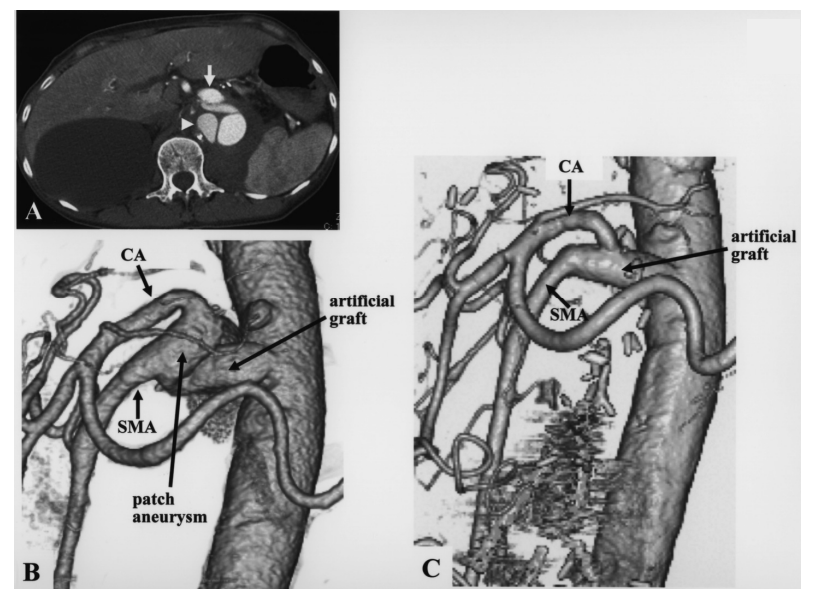

Figure 1. (a) Preoperative computed tomographic (CT) scan demonstrated a visceral patch aneurysm (arrow) and an extravascular pooling (arrow head); (b) Preoperative CT angiogram showed extravascular leakage at the anastomotic site of the artificial branched graft and a visceral patch aneurysm involving the celiac axis (CA) and superior mesenteric artery (SMA); (c) Postoperative CT angiogram demonstrated absence of visceral patch aneurysm, extravascular leakage and patency of the CA and SMA.

partial detachment of the suture. The SMA was reanastomosed to the branch graft directly. The CA was reattached to the side hole of the prosthetic branch graft with an end-to-side anastomotic technique. Postoperative CT scan showed that the hematoma in the retroperitoneal space regressed and extravascular leakage disappeared (Figure 1(c)). One month later, the patient presented with severe back pain again. CT scan demonstrated an aortic pseudoaneurysm involving reconstructed ICA and a huge extravascular pooling (Figure 2(a)). Under general anesthesia, the patent T8 ICA was embolized with coils to prevent type II endoleak and then endovascular repair using a $31 \times 150 \mathrm{~mm}$ Gore TAG Thoracic Endograft (W. L. Gore and Associates, Inc. Flagstaff, Arizona) was performed. The distal end of the endograft was positioned just above the first branch of the aortic graft. After the stent-graft deployment, CT angiogram confirmed patency of the first branch and the absence of endoleak (Figure 2(b)). One month later, the patient presented with severe back pain again. CT scan demonstrated an extravascular leakage from the anastomotic site of the SMA that was attached to the branched graft (Figure 3(a)). Measures were taken on an emergency basis. The proximal end of the CA was embolized with coils. Then, a 10 $\times 60 \mathrm{~mm}$ fully covered Niti-S stent (Taewoong Medical, Seoul, South Korea) was deployed to cover the anastomotic site of the SMA. Postoperative CT angiogram showed absence of endoleak and perfusion of the distal CA (Figure 3(b)). Though the precise causes of repeated anastomotic failures were unclear, it might be related to the weakness of aortic tissue. One year later CT scan re-

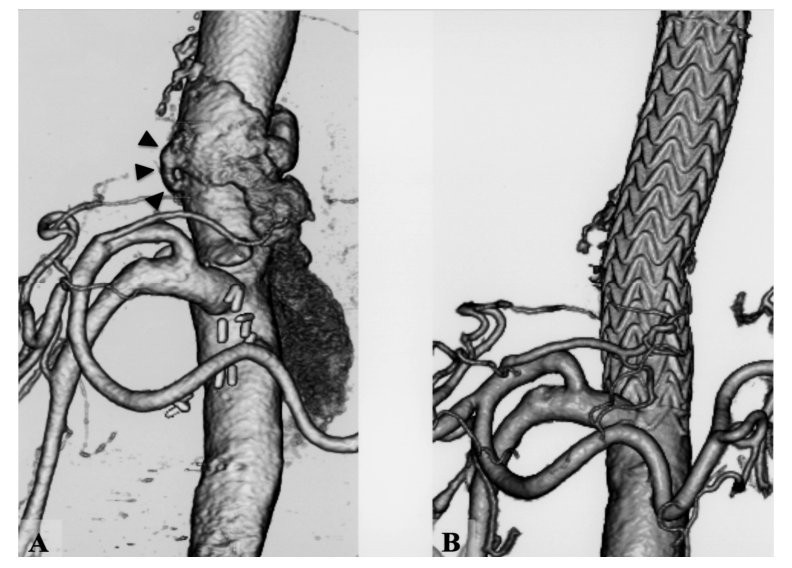

Figure 2. (a) Preoperative computed tomographic (CT) angiogram showed an aortic patch aneurysm (arrow heads) involving an intercostal artery and a huge extravascular pooling; (b) Postoperative CT angiogram demonstrated complete exclusion with a TAG stent-graft of the intercostal artery patch without endoleak.
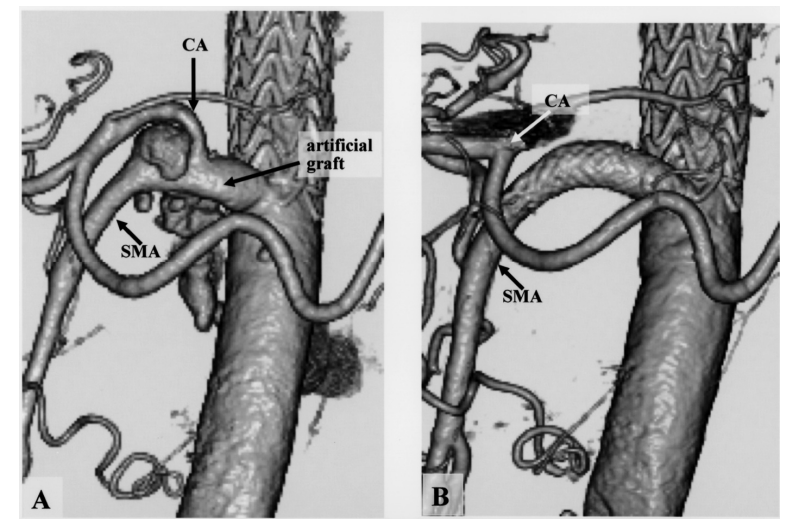

Figure 3. (a) Preoperative computed tomographic (CT) angiogram showed an anastomotic pseudoaneurysm of the artificial branch graft and the superior mesenteric artery (SMA); (b) Postoperative CT angiogram showed absence of the pseudoaneurysm and patency of distal side of the celiac axis $(C A)$.

vealed no evidence of recurrent aneurysm, stent migration or endoleak.

\section{Discussion}

Paraplegia resulting from spinal cord ischemia during TAAA surgery is a dreadful complication. Various methods have been used for the prevention of ischemic spinal cord injury [10-12]. Several reconstruction techniques have been reported for the ICA and visceral arteries. An island reconstruction is the simplest because of the small number of anastomoses and the short time required for revascularization. Although an island technique is used whenever the arteries are found reasonably healthy, reconstructed aortic patch may gradually dilate and transform into aneurysms. Separate revascularization of the 
ICA or visceral arteries requires more anastomoses than an island technique, increasing the risk of bleeding and extending operative time. However, the small size of the residual aorta means the risk of aneurysm formation at the site of reconstruction is low and the prospect of reoperation is negligible. Kulik and colleagues reviewed their experience of 155 patients who underwent descending or TAAA repair; the incidence of ICA patch aneurysm development was 7.1\% [13]. Dardik and coworkers reviewed their experience of 107 patients undergoing TAAA repair with reconstruction of visceral arteries and identified aortic patch aneurysms in 8 (7.5\%) patients [2]. Tshomba and associates reviewed their experience with TAAA operations in 182 patients and reported that 6 (3.3\%) patients had visceral artery aneurysms, and 1 (0.5\%) patient had a pseudoaneurysm [4]. Heretofore, the treatment of aortic patch aneurysm was performed by open surgery via redo thoraco-phrenico-laparotomy. A simple laparotomy as in our case might be a good approach for the restricted lesions. The successful cases of aortic patch aneurysm repair under redo open surgery via a thoracophrenico-laparotomy are reported in several articles $[1,3,14$, 15]. However, the procedure has carried a high mortality and morbidity, because the thoracic or retroperitoneal redissection is challenging due to the dense adhesion making the aneurysm difficult to re-dissect and increasing the risk of major bleeding. An EVSG technique for aortic patch aneurysm might reduce the operative risk and has been recently reported in many studies [4-9,13]. It is comparatively easier to repair an ICA patch aneurysm from a visceral patch aneurysm with an endovascular procedure. All it requires is the deployment of a simple stent-graft in the previous aortic graft. Though patent Th8 segmental artery was embolized in our case, only for the prevention of extravasuclar leakage this embolization might not be necessary. Fortunately the patient suffered no neurological deficits. A hybrid technique or a surgeon-modified endograft is used for the treatment of a visceral artery patch aneurysm [5-8], because the visceral arteries have to be reconstructed to avoid ischemia to major abdominal viscera. We could treat patch aneurysm and pseudoaneurysm of the visceral artery through a small laparotomy or with a simple stent-graft technique. This was possible because we reconstructed the visceral arteries using a branched aortic graft during the initial TAAA operation. Therefore, despite some of its drawbacks that include long operative time, bleeding, morbidity and mortality, we think that the visceral arteries should be individually reconstructed with a branched graft during TAAA surgery.

\section{REFERENCES}

[1] T. P. Carrel and C. Signer, "Separate Revascularization of the Visceral Arteries in Thoracoabdominal Aneurysm Repair,” The Annals of Thoracic Surgery, Vol. 68, No. 2, 1999, pp. 573-575. doi:10.1016/S0003-4975(99)00574-3

[2] A. Dardik, B. A. Perler, G. S. Roseborough and G. S. Williams, "Aneurysmal Expansion of the Visceral Patch after Thoracoabdominal Aortic Replacement: An Argument for Limiting Patch Size?” Journal of Vascular Surgery, Vol. 34, No. 3, 2001, pp. 405-410. doi:10.1067/mva.2001.117149

[3] K. Suzuki, T. Kazui, T. Ohno, K. Sugiki, H. Doi and Y. Ohkawa, "Re-Reconstruction of Visceral Arteries with Thoracoabdominal Aortic Replacement Using a Branched Graft," Japanese Journal of Thoracic and Cardiovascular Surgery, Vol. 53, No. 4, 2005, pp. 217-219. doi:10.1007/s11748-005-0110-y

[4] Y. Tshomba, L. Bertoglio, E. M. Marone, G. Melissano and R. Chiesa, "Visceral Aortic Patch Aneurysm after Thoracoabdominal Aortic Repair: Conventional vs Hybrid Treatment," Journal of Vascular Surgery, Vol. 48, No. 5, 2008, pp. 1083-1091.

doi:10.1016/j.jvs.2008.05.079

[5] A. M. Bakken, C. D. Protack, D. L. Waldman and M. G. Davies, "Hybrid Debranching-Endovascular Repair of Visceral Patch Aneurysm after Thoracoabdominal Aneurysm Repair," Vascular and Endovascular Surgery, Vol. 41, No. 3, 2007, pp. 249-253. doi:10.1177/1538574407301432

[6] D. J. Adam, M. Berce, D. E. Hartley, D. A. Robinson and J. L. Anderson, "Repair of Recurrent Visceral Aortic Patch Aneurysm after Thoracoabdominal Aortic Aneurysm Repair with a Branched Endovascular Stent Graft,” Journal of Vascular Surgery, Vol. 45, No. 1, 2007, pp. 183-185. doi:10.1016/j.jvs.2006.08.023

[7] L. M. Reilly and T. A. Chuter, "Endovascular Repair of Thocacoabdominal Aneurysms: Design Options, Device Construct, Patient Selection and Complications,” Journal of Vascular Surgery, Vol. 50, 2009, pp. 447-460.

[8] J. Jim, L. A. Sanchez and B. G. Rubin, "Use of a Surgeon-Modified Branched Thoracic Endograft to Preserve an Aortorenal Bypass during Treatment of an Intercostal Patch Aneurysm,” Journal of Vascular Surgery, Vol. 52, No. 3, 2010, pp. 730-733. doi:10.1016/j.jvs.2010.03.062

[9] S. Schwill, S. A. LeMaire, S. Y. Green, F. G. Bakaeen and J. S. Coselli, "Endovascular Repair of Thoracic Aortic Pseudoaneurysms and Patch Aneurysms," Journal of Vascular Surgery, Vol. 52, No. 4, 2010, pp. 1034-1037. doi:10.1016/j.jvs.2010.04.078

[10] H. J. Safi, C. C. Miller III, T. T. T. Huynh, A. L. Estrera, E. E. Porat, A. N. Winnerkvist, et al., "Distal Aortic Perfusion and Cerebrospinal Fluid Drainage for Thoracoabdominal and Descending Thoracic Aortic Repair," Annals of Surgery, Vol. 238, No. 3, 2003, pp. 372-381.

[11] N. T. Kouchoukos, B. B. Daily and C. K. Rokkas, "Hypothermic Bypass and Circulatory Arrest for Operations on the Descending Thoracic and Thoracoabdominal Aorta,” The Annals of Thoracic Surgery, Vol. 60, No. 1, 1995, pp. 67-77.

[12] K. Suzuki, T. Kazui, H. Terada, K. Umemura, Y. Ikeda, A. H. Bashar, et al., "Experimental Study on the Protec- 
tive Effects of Edaravone against Ischemic Spinal Cord Injury," The Journal of Thoracic and Cardiovascular Surgery, Vol. 130, No. 6, 2005, pp. 1586-1592.

doi:10.1016/j.jtcvs.2005.08.049

[13] A. Kulik, B. T. Allen and N. T. Kouchoukos, "Incidence and Management of Intercostal Patch Aneurysms after Repair of Thoracoabdominal Aortic Aneurysms," The Journal of Thoracic and Cardiovascular Surgery, Vol. 138, No. 2, 2009, pp. 352-358.

doi:10.1016/j.jtcvs.2009.01.005

[14] R. R. Dias, J. S. Coselli, N. A. Stolf, A. R. Dias, C. Mady and S. A. Oliveira, "Aneurysmal Dilation of the Reimplant Segment of the Visceral Vessels after Thoracoabdominal Aneurysm Correction," Arquivos Brasileiros de Cardiologia, Vol. 81, No. 3, 2003, pp. 273-278. doi:10.1590/S0066-782X2003001100007

[15] J. Kokotsakis, G. Lazopoulos, H. Ashrafian, P. Misthos, T. Athanasiou and A. Lioulias, "Thoracoabdominal Aneurysm Repair Using a Four-Branched Thoracoabdominal Graft: A Case Series,” Cases Journal, Vol. 2, No. 1, 2009, p. 7144. $\underline{\text { doi:10.4076/1757-1626-2-7144 }}$ 